\title{
QSAR Analysis of Rocaglamide Derivatives Cytotoxic Activities Using LFER Hansch Model
}

\author{
Firdayani $^{1}$, Susi Kusumaningrum ${ }^{1}$, Doddy Irawan Setyo Utomo ${ }^{1}$, \\ Agung Eru Wibowo ${ }^{1}$, Chaidir ${ }^{1}$ \\ ${ }^{1}$ Center of Pharmaceutical and Medical Technology \\ Agency on Assessment and Application of Technology (BPPT) \\ BPPT $2^{\text {nd }}$ Building $15^{\text {th }}$ Floor, Jl. MH. Thamrin 8 JAKARTA
}

\begin{abstract}
Abstact
Rocaglamide derivatives are the compounds which have featuring cyclopenta[b]tetrahydrobenzofuran skeleton. Until now it includes more than 50 naturally occurring derivatives. They were chosen to be interesting candidates for possible therapeutic agents primarily in the field of cancer chemotherapy due to their cytotoxic activities data against various cancer cells. A quantitative structure activity relationship (QSAR) studies were done to investigate physicochemical properties of molecule which contribute to their activities. Series of rocaglamide derivatives have been used and analyzed using linear free energy regression Hansch model for their cytotoxic activities against MONO-MAC-6 leukemia cells, RAJI lymphoma cells and MEL-JUSO melanoma cells. Results showed that the best QSAR equations were revealed involving $C$ Log $P$ and CMR parameters with nonlinear regression relationships in cytotoxic activities of rocaglamide derivatives against cancer cells above.
\end{abstract}

Keywords: QSAR, Rocaglamide, LFER Hansch

\section{INTRODUCTION}

Natural products are rich source of biologically active compound such as Rocaglamide. This compound and its naturally occurring congeners are tetrahydrobenzofurans that occur exclusively in member of genus Aglaia. These natural products are believed to have strong insecticidal activity. Recently, Rocaglamide derivatives have also been found to have an inhibitory effect on the activity of the proinflammatory transcription factor NF- $\mathrm{kB}$. They are potent immunosuppressive phytochemicals that inhibit cytokine gene expression at the transcription level such as IFN- $\gamma$, TNF- $\alpha$, IL-2 and IL-4 production in peripheral blood $\mathrm{T}$ cells at nanomolar concentration.

It is suggested that rocaglamide have potential role in the development of drugs against inflammation and graft rejection after transplantation. Rocaglamide derivatives pronounced cytostatic activity against human cancer cell lines and also able to elicit apoptosis in resistant tumor cells (Proksch, 2005). These data make these compounds interesting candidates for possible therapeutic agents primarily in the field of cancer chemotherapy.

Quantitative structure activity relationship (QSAR) is an important part of drug design, in an attempt to get a new drug with greater activity, higher selectivity, toxicity or side effects as small as possible and greater comfort.

In addition, by using the QSAR model will be more cost-saving or more economical, because to get new drugs with the desired activity, try to factor suppressed as small as possible so that the path becomes shorter synthesis (Siswandono, 2000).

\footnotetext{
*Corresponding author e-mail: yanifirda@yahoo.co.id
} 
QSAR method can be generally defined as an application of mathematical and statistical methods to the problem of finding empirical relationships (QSAR models) of the form $P i=k^{\wedge}(D 1, D 2, \ldots D n)$, where $\mathrm{Pi}$ are biological activities (or other properties of interest) of molecules, $D 1, D 2, \ldots, D n$ are calculated (or, sometimes, experimentally measured) structural properties (molecular descriptors) of compounds, and $k^{\wedge}$ is some empirically established mathematical transformation that should be applied to descriptors to calculate the property values for all molecules. The relationship between values of descriptors D and target properties $\mathrm{P}$ can be linear or non-linear.

The example of the former relationship is given by multiple linear regression (MLR) common to Hansch QSAR approach, where target property can be predicted directly from the descriptor values. The goal of QSAR modeling is to establish a trend in the descriptor values, which parallels the trend in biological activity. In essence, all QSAR approaches imply, directly or indirectly, a simple similarity principle, which for a long time has provided a foundation for the experimental medicinal chemistry: compounds with similar structures are expected to have similar biological activities. QSAR approach attemps to identify and quantify the physicochemical properties of a drug and to see whether any of these properties has an effect on the drug's biological activity. By quantifying physicochemical properties, it should be possible to calculate in advance what the biological activity of a novel analogue might be. (Patrick, 2005)

Hydrophobic character of a drug is crucial to how easily it crosses cell membrane and may also be important in receptor interactions. Changing substituents on a drug may have significant effect on its hydrophobic character and hence its biological activity. This reflects that the drug must pass through the hydrophobic barrier such as cell membranes in order to reach the target. Although there is no barrier that must be passed (e.g. in vitro studies), drug compound must interact with a target system such as enzymes or receptors in which the binding site is usually active also hydrophobic. In other hand, the bulk, size and shape of a drug will influence how easily it can approach and interact with a binding site. (Patrick, 2005).

There are several models of QSAR model include the Free-Wilson Model, Linear Free Energy Regression (LFER) Hansch Model, approach to quantum mechanics and molecular connectivity. In this research, LFER Hansch approach model will be used in the analysis of quantitative structure activity relationship rocaglamide derivatives to determine the physicochemical properties that influence on cytotoxic activity against various cancer cells such as MONO-MAC-6, MEL-JUSO and RAJI.

\section{METHODS}

In this study we used Notebook with specification: Intel core $\mathrm{i} 3$, DDR3 2GB + $1 \mathrm{~GB}$, HDD $320 \mathrm{~GB}$ and Windows 7 operation system. The most stable structure of rocaglamide derivatives were drawn and then hydrophobicity $(\log P$ and $C \log P$ ) and steric (MR and $C M R$ ) propertie values from each compound were obtained theoretically using Chembiodraw software program.

$I C_{50}$ data of rocaglamide derivatives collected from Bohnenstangel (2000). $C$ is the nanomolar concentration that inhibit 50\% growth of cancer cell; $\log 1 / C$ is the subsequent dependent variable that defines the biological parameter for QSAR development.

The properties owned by their respective compounds were linked with cytotoxic activity data. $C \log P$ is the calculated partition coefficient of a compound in n-octanol/water and is a measure of its hydrophobicity. $C M R$ is the calculated Molar refractivity (MR) for the whole molecule. Then, we determined the best linear or nonlinear regression equation between the properties of hydrophobic and steric with cytotoxic activity data from Bohmestangel (2000) using SPSS statistic program.

\section{RESULTS}

The structure of Rocaglamide derivatives were investigated for QSAR analysis in this study are shown figure 1 . 
<smiles>[R]c1cc([C@]23Oc4cc(OC)cc(OC)c4[C@@]2([R8])[C@H]([R8])[C@H]([13CH2])[C@H]3c2ccccc2)ccc1OC</smiles>

[1 -11]

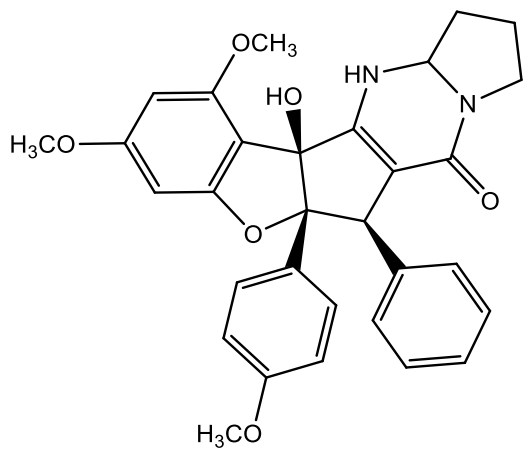

[12]

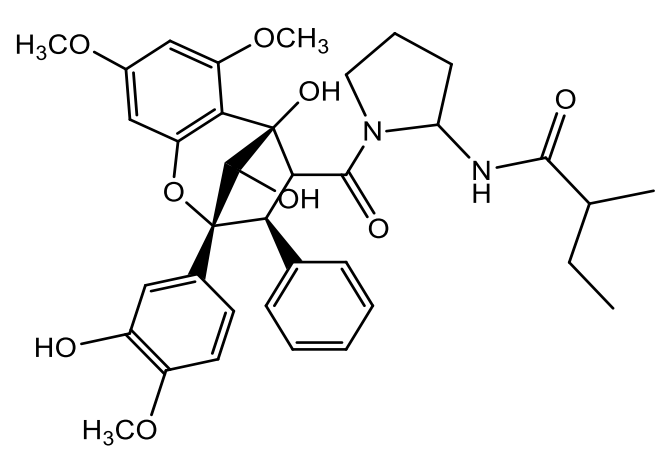

[13]

Fig I. Structure of Rocaglamide Derivatives

Table I. Side chain substituen of Rocaglamide derivatives [ I- I I]

\begin{tabular}{|c|c|c|c|c|}
\hline Rocaglamide & $\mathbf{R}_{\mathbf{I}}$ & $\mathbf{R}_{\mathbf{2}}$ & $\mathbf{R}_{\mathbf{3}}$ & $\mathbf{R}_{\mathbf{4}}$ \\
\hline$[\mathrm{I}]$ & $\mathrm{OH}$ & $\mathrm{OH}$ & $\mathrm{CONH}_{2}$ & $\bar{H}$ \\
\hline [2] & $\mathrm{OH}$ & $\mathrm{OH}$ & $\mathrm{CONH}_{2}$ & $\mathrm{OH}$ \\
\hline [3] & $\mathrm{OH}$ & $\mathrm{OH}$ & $\mathrm{CONHCH}_{4}$ & $\mathrm{H}$ \\
\hline [4] & $\mathrm{OH}$ & $\mathrm{OH}$ & $\mathrm{CONHCH}_{3}$ & $\mathrm{OH}$ \\
\hline [5] & $\mathrm{OH}$ & $\mathrm{OCOCH}_{3}$ & $\mathrm{CONHCH}_{3}$ & $\mathrm{OH}$ \\
\hline [6] & $\mathrm{OH}$ & $\mathrm{OH}$ & $\mathrm{CON}\left(\mathrm{CH}_{3}\right)_{2}$ & $\mathrm{H}$ \\
\hline [7] & $\mathrm{OH}$ & $\mathrm{OH}$ & $\mathrm{CON}\left(\mathrm{CH}_{3}\right)_{2}$ & $\mathrm{OH}$ \\
\hline [8] & $\mathrm{OH}$ & $\mathrm{OH}$ & $\mathrm{CON}\left(\mathrm{CH}_{3}\right)_{2}$ & $\mathrm{OCH}_{3}$ \\
\hline [9] & $\mathrm{OH}$ & $\mathrm{OCOCH}_{3}$ & $\mathrm{CON}\left(\mathrm{CH}_{3}\right)_{2}$ & $\mathrm{OH}$ \\
\hline [10] & $\mathrm{OH}$ & $\mathrm{OH}$ & $\mathrm{CONH}\left(\mathrm{CH}_{2}\right)_{4} \mathrm{OH}$ & $\mathrm{H}$ \\
\hline$[11]$ & $\mathrm{OH}$ & $\mathrm{OH}$ & $\mathrm{COOCH}_{3}$ & $\mathrm{OH}$ \\
\hline
\end{tabular}


Biological activities data and Physicochemical Properties value of Rocaglamide derivatives are shown in Table 2.

Table 2. $C \log P$ and $C M R$ value and cytotoxic data of Rocaglamide derivatives

\begin{tabular}{|c|c|c|c|c|c|c|c|c|}
\hline \multirow{2}{*}{ Rocaglamide } & \multirow[b]{2}{*}{$C \log P$} & \multirow[b]{2}{*}{$C M R$} & \multicolumn{2}{|c|}{ MONO-MAC-6 } & \multicolumn{2}{|c|}{ MEL-JUSO } & \multicolumn{2}{|c|}{ RAJI } \\
\hline & & & $I C_{50}(\mathrm{~nm})$ & $\log I / I C_{50}$ & $I C_{50}(\mathrm{~nm})$ & $\log I / I C_{50}$ & $I C_{50}(\mathrm{~nm})$ & $\log I / I C_{50}$ \\
\hline$[\mathrm{I}]$ & 2.1476 & 13.164 & 4 & -0.60 & 13 & $-I . I I$ & & \\
\hline [2] & 2.1244 & 13.006 & 37 & -1.57 & 51 & -1.71 & & \\
\hline [3] & 2.0814 & 13.317 & 22 & -1.35 & 31 & -1.49 & 35 & -1.54 \\
\hline [4] & 2.4916 & 13.470 & 105 & -2.02 & 81 & -1.91 & & \\
\hline [5] & 3.3388 & 14.434 & 188 & -2.28 & 164 & -2.21 & & \\
\hline [6] & 2.5782 & $|3.78|$ & 32 & -1.52 & 18 & -1.25 & 32 & -1.20 \\
\hline [7] & 2.4782 & 13.934 & 54 & -1.74 & 40 & -1.60 & 175 & -2.24 \\
\hline [8] & 3.4642 & 14.398 & 172 & -2.22 & 92 & -1.96 & 187 & -2.25 \\
\hline [9] & 3.8356 & 14.897 & 82 & -1.92 & 57 & -1.77 & 178 & -2.25 \\
\hline$[10]$ & 1.6814 & 14.862 & 24 & -1.39 & 22 & -1.35 & 18 & -1.26 \\
\hline [II] & 3.172 & 13.255 & 242 & -2.4 & 138 & -2.15 & 197 & -2.3 \\
\hline [12] & 4.5968 & $|4.53|$ & 40 & -1.6 & 32 & -1.52 & & \\
\hline [13] & 2.9222 & 18.182 & 4600 & -3.7 & 4600 & -3.7 & & \\
\hline
\end{tabular}

\section{Cytotoxic activity against MONO-MAC-6 leukemia cells}

Based on biological activity data of Rocaglamide derivatives against MONO-MAC-6 cells which derived from dissertation of Bohnenstangel (2000) and physicochemical properties used to derived QSAR analysis, it is determined the quotations above.

a. a linear regression model

$\log 1 / C=-0.099 C \log P-0.375 C M R+3.75$

$n=13 ; r=0.74 ; r^{2}=0.55 ; F=6.029 ; s=0.54$

(1)

\section{b. a nonlinear regression model}

$\log 1 / C=-0.17(C \log P)^{2}+0.57 C \log P+0.11 C M R-3.48$

$n=13 ; \quad r=0.82 ; \quad r^{2}=0.67 ; \quad F=6.126 ; \quad s=0.48$

\section{Cytotoxic activity against RAJI lymphoma cells}

Based on biological activity data of Rocaglamide derivatives against RAJI lymphoma cells from Bohnenstangel (2000) and physicochemical properties used to derived QSAR analysis, it is determined the quotations above.
1. a linear regression model

$\log 1 / C=-0.57 C C \log P-0.098 C M R-1.64$

$n=7 ; \quad r=0.85 ; \quad r^{2}=0.72 ; \quad F=5.084 ; \quad s=0.33$

2. a nonlinear regression model

$\log 1 / C=0.01(C \log P)^{2}-0.614 C \log P+0.1 C M R-1.6$

$n=7 ; \quad r=0.85 ; \quad r^{2}=0.72 ; \quad F=2.56 ; \quad s=0.38$

(4)

\section{Cytotoxic activity against MEL-JUSO melanoma cells}

Based on biological activity data of Rocaglamide derivatives against MEL-JUSO melanoma cells from Dissertation of Bohnenstangel (2000) and physicochemical properties used to derived QSAR analysis, it is determined the quotations above.

\section{1. a linear regression model}

$\log 1 / C=0.013 C \log P-0.363 C M R+3.314$

$n=13 ; \quad r=0.762 ; \quad r^{2}=0.58 ; \quad F=6.904 s=0.46$ 
2. a nonlinear regression model

$\log 1 / \mathrm{C}=-0.2(\mathrm{C} \log \mathrm{P})^{2}+0.81 \mathrm{C} \log \mathrm{P}+2.1 \mathrm{CMR}-5.22$

$n=13 ; \quad r=0.89 ; \quad r^{2}=0.80 ; \quad F=12.067 ; \quad s=0.33$

\section{DISCUSSION}

A quantitative structure-activity relationship (QSAR) related numerical properties of the molecular structure to its activity by a mathematical model. In drug design, QSAR methods have often been used to consider qualities beyond in vitro potency. A structure-activity study can help to dicide which features of a molecule give rise to its overall activity and help to modified compounds with enhance properties.

The most widely used technique for deriving QSAR equations is linear regression, which uses least-quares fitting to find the best combination of coefficients in the QSAR equation. In QSAR equation, $n$ is the number of data points, $r$ is the correlation coefficient between observed values of the dependent and the values calculated from the equation, $r^{2}$ is the square of the correlation coefficient and represent the goodness of fit, $F$ is the Fischer statistics (Fischer ratio) and $s$ is the standard deviation. The F-value is actually the ratio between explained and unexplained variance for a given number of degree of freedom. Thus, it indicates a true relationship, or the significance level for the MRL models.

Based on QSAR analysis on cytotoxic activity against three types of cancer cell lines here, it is indicated that there is a relationship between hydrophobicity $\left(\begin{array}{lll}C & \log & P\end{array}\right)$ and steric $(C M R)$ properties with biological activity. The hydrophobic character of a substances is crucial to how easily it crosses cell membrane and may also be important in receptor interactions. Changing substituent on a drug may well have significant effect on its hydrophobic character and hence its biological activity. It is generally found that increasing the hydophobicity of a lead compound results in an increase in biological activity. This reflect the fact that drugs have to cross hydrophobic barriers such as cell membranes in order to reach their target. Even if no barriers are to be crossed (like in vitro studies), the drug has to interact with a target system such as an enzyme or receptor where the binding site is usually hydrophobic. However, the drug must be sufficiently lipophilic (lipid loving) to partition in the membrane but no so lipophilic that it stay there. This explains the parabolic dependence or non linear correlation of the activity upon $\mathrm{Clog} P$

The bulk, size and shape of a drug will influence how easily it can approach and interact with a binding site. A bulky substituent may act like a shield and hinder the ideal interaction between a drug and its binding site. Alternatively, a bulky substituent may help to orientate a drug properly for maximum binding and increase activity (Patrick, 2005; Leach, 2001)

The relation on physicochemical properties with cytotoxic activity against MONO-MAC-6 and MEL-JUSO occur more likely in non-linear regression model. They are indicated with statistical parameters of equation (2) showed better results than equation (1) neither does the equation (6) compared with equation (5). Whereas relation on physicochemical properties with cytotoxic activity against RAJI cells were more likely to linear regression relationship, although equation (3) and (4) showed similar parameter $r$ and $r^{2}$ values but F-value on linear regression is greater than nonlinear regression.

\section{CONCLUSION}

In summary, we have utilized QSAR as a tool to determine the physicochemical parameters that influence induced cytotoxicity of rocaglamide derivatives. This study had conclusion that $C \log P$ and $C M R$ of rocaglamide related with its biological activity.

\section{REFERENCES}

Bohnenstengel, F., 2000., Inhaltsstoffe aus tropischen Meliaceen der Gattungen Aglaia und Melia, dissertation, Bayerischen JuliusMaximilians- Universität Wurzburg.

Hansch, C., R.P Verma, 2008., A QSAR study for the cytotoxic activities of taxoids Against macrophage (MФ)-like cells, Euro, Journal of Med. Chem, 44, 274-279.

Kim, S., Salim, A.A., Swanson, S.M. and Kinghorn, A.D., 2006, Potential of Cyclopenta[b]benzofurans from Aglaia Species in Cancer Chemotherapy, Anticancer Agents in Med. Chem., 6, 319-345. 
Leach, A.R., 200I, Molecular Modelling Principles and Application, $2^{\text {nd }}$ Ed, Pearson Prentice Hall, England, pp 640-7/8.

Patrick, G.L., 2005, An Introduction to Medicinal Chemistry, 3rd Ed, Oxford Univ. Press, New York, Pp 27I-296.

Proksch, P., Edrada, R., Ebel, R., Bohnestengel, F.I, and Nugroho, B.W., 200I, Chemistry and Biological Activity of Rocaglamide Derivatives and Related Compounds in Aglaia Species (Meliaceae), Curr Org. Chem., 5, 923-938.

Proksch, P., Giaisi, M., Treiber, M.K., Palfi, K., Merling, A., Spring, H., Krammer, P.H. and Weberl, M.L., 2005, Rocaglamide
Derivatives Are Immunosuppressive Phytochemicals That Target NF-AT Activity in T Cells, The Journal of Immunology, I74, 7075-7084.

Tropsha, A and Golbraikh, A., 2007, Predictive QSAR Modeling Workflow, Model Applicability Domains, and Virtual Screening. Current Pharmaceutical Design, I3, 3494-3504. 